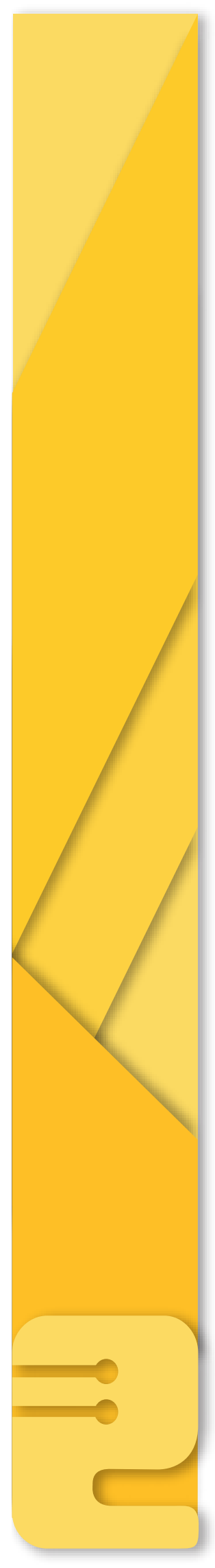

\title{
LA INCORPORACIÓN DE LAS TECNOLOGÍAS EDUCATIVAS EN LOS PROCESOS DE FORMACIÓN INICIAL DE DOCENTES DEL NIVEL DE EDUCACIÓN BÁSICA EN LA UNIVERSIDAD CATÓLICA DE EL SALVADOR ${ }^{1}$
}

\author{
INCLUSION OF EDUCATIVE TECHNOLOGIES IN TRAINING PROCESSES FOR TEACHERS OF \\ ELEMENTARY SCHOOL LEVEL IN UNIVERSIDAD CATÓLICA DE EL SALVADOR \\ A INCORPORAÇÃO DAS TECNOLOGIAS EDUCATIVAS NOS PROCESSOS DE FORMAÇÃO \\ INICIAL DE DOCENTES DO NÍVEL DE EDUCAÇÃO BÁSICA NA UNIVERSIDAD CATÓLICA DE \\ EL SALVADOR
}

Nery Francisco Herrera Pineda², Antonia Larissa Hernández Monterrosa ${ }^{3}$

Fecha de Recibido: Agosto 01 de 2016 | Fecha de Aprobado: Septiembre 22 de 2016

\section{Resumen}

El objetivo principal de esta investigación fue incorporar tecnología educativa en el proceso de enseñanza aprendizaje de los estudiantes de la carrera académica de Profesorado de Educación Básica para primer y segundo ciclo. La metodología implementada fue de tipo descriptiva - evaluativa y se hace énfasis en cómo en las asignaturas de esta carrera se incorpora este elemento. Además, se evaluó la asimilación y aplicación que tuvieron los docentes y los estudiantes con respecto a las herramientas tecnológicas en el desarrollo de las clases. Se concluye que al realizar innovación en el proceso de enseñanza aprendizaje con tecnología se puede lograr que los estudiantes obtengan un aprendizaje más significativo, y también, que la preparación de los formadores que tienen a su cargo grupos de futuros formadores, deben actualizar sus conocimientos en el aspecto tecnológico para que su práctica pedagógica sea más eficiente.

Palabras clave: Educación básica, tecnología, metodología, recursos tecnológicos, formación docente, competencia digital.

1 Artículo de reporte de caso.

2 Máster en Profesionalización de la Docencia Superior y Máster en Métodos y técnicas de Investigación Social, Universidad Católica de El Salvador. Correo electrónico: nery.herrera@catolica.edu.sv

3 Máster en Profesionalización de la Docencia Superior y Licenciatura en Ciencias de la Educación con especialidad en Educación Parvularia, Universidad Católica de El Salvador, Red de investigadores del observatorio de la Diversidad Religiosa y de las Culturas en América Latina y El Caribe. Correo electrónico: larissa.hernandez@catolica.edu.sv 


\section{Abstract}

The main objective of this study was to incorporate educative technology in teaching-learning process of students who study the elementary school teaching professional program in first and second level. The methodology applied to this research was based on descriptive - evaluative model and it is focused on how to implement this method into the subjects of this program. Moreover, the adapting of teachers and students was evaluated in regards to thechnolgical tools used during tha class' development. In conclusion, the use of innovative technology within the teaching-learning process assure that students attain a more significant learning. In addition, the training of formers who are responsible for future teachers' education must update their knowledge about technology to improve their pedagogical practice.

Keywords: Elementary school, technology, methodology, technological resources, teaching training, digital competence.

\section{Resumo}

O objetivo principal desta investigação foi incorporar tecnologia educativa no processo de ensino aprendizagem dos estudantes da carreira acadêmica de profesorado de educação básica para primeiro e segundo ciclo. A metodologia implementada foi de tipo descritiva - avaliativa e faz-se ênfase em como nas matérias desta carreira se incorpora este elemento. Além disso, avaliou-se a assimilação e aplicativo que tiveram os docentes e os estudantes com respeito às ferramentas tecnológicas no desenvolvimento das classes. Conclui-se que ao realizar inovação no processo de ensino aprendizagem com tecnologia pode ser conseguido que os estudantes obtenham uma aprendizagem mais significativa, e também, que a preparação dos formadores que têm a seu cargo grupos de futuros formadores, devem actualizar seus conhecimentos no aspecto tecnológico para que sua prática pedagógica seja mais eficiente.

Palavras chave: Educação básica, tecnologia, metodologia, recursos tecnológicos, formação docente, concorrência digital. 


\section{INTRODUCCIÓN}

Con esta investigación se realizó una reflexión y análisis de la necesidad de incorporar la tecnología al proceso de enseñanza aprendizaje de los futuros profesores de Educación Básica para primer y segundo ciclo; y de cómo los docentes, en su mayoría inmigrantes digitales, término que según Tochero (2015) hace referencia a "quienes han conocido la época previa y se han ido introduciendo en la sociedad red a medida que las TIC iban llegando a sus vidas" (p.1); que formaron parte del estudio fueron capacitados en función de la planeación y aplicación didáctica de las tecnologías educativas a nivel de Educación Superior, logrando de esta manera crear y recrear ambientes adecuados en los cuales los estudiantes acomoden el uso de estos medios en la concreción de su aprendizaje, además de mejorar sus habilidades y destrezas con este tipo de recurso.

Se refleja en esta investigación, que la presencia de éstas metodologías de enseñanza no todos los docentes la utilizan, por varios factores: algunos porque no son especialistas en el área; por lo tanto, no poseen los conocimientos informáticos suficientes para sentirse cómodos empleándolas en su práctica docente, quedándose en lo tradicional, es decir, usar solamente la pizarra o en la institución no tienen los recursos tecnológicos suficientes. Para el caso de los docentes catedráticos que incorporan a sus clases recursos y herramientas tecnológicas, hacen la diferencia en el sistema educativo, en el sentido que imparten conocimientos extra a los estudiantes para que la clase sea desarrollada con elementos que provoquen interés en los estudiantes, tomando en cuenta que son nativos digitales y es una generación que tiene habilidades cognitivas diferentes a sus predecesores, por tal razón.

\section{ANTECEDENTES}

En el ámbito de la Educación Superior a nivel mundial, el uso de las tecnologías se ha convertido en uno de los requerimientos básicos para el desarrollo de los procesos de enseñanza-aprendizaje, ya que el rápido avance tecnológico se transporta a estar inmersos en un acelerado cambio de tal manera que "Las Tecnologías de la Información y la Comunicación (TIC) se han convertido en un elemento esencial en los nuevos espacios de interacción del ser humano" (Nakano, Garret, Vásquez \& Águeda, 2014, p. 65). El reto surge para los docentes encargados de la enseñanza, quienes deben incluir en el currículo los diferentes medios tecnológicos durante el desarrollo de la clase, brindando así un soporte a las actividades de enseñanza-aprendizaje que buscan el desarrollo de capacidades y habilidades en los estudiantes para cuando estén ejerciendo como profesores en las escuelas.

Por otra parte, la incorporación de recursos tecnológicos ayuda en gran medida a innovar la práctica pedagógica, ya que estos elementos tienen funciones específicas que ayudan en el proceso de enseñanza-aprendizaje. Además, al hablar hoy de tecnologías lleva inexorablemente a pensar tal como lo plantea Scolari (2011): "En las computadoras, en Internet y también en una amplia nueva generación de dispositivos móviles y de aplicaciones en red que plantean nuevos escenarios" (p.8). Nuevos escenarios que invitan a explorar esos recursos que ayudan a ampliar los conocimientos y que según Cabero (2007), se encuentran: "La función motivadora, función estructuradora, función informativa o de portadores de contenido y función innovadora “(pp. 57-58); por lo tanto, al incorporarlos, el proceso educativo se mejorará de manera eficiente, ya que los recursos en la enseñanza son fundamentalmente necesarios. Blanco, Rico y Pino (2009) consideran que estos recursos son importantes porque permiten “(...) ilustrar, motivar, informar, transmitir información, configurar el conocimiento y promueven la discusión" (p. 1210); enriqueciendo y modernizando así el proceso de enseñanza, es de tomar en cuenta que los estudiantes -nativos digitales- de esta época les gusta que las clases sean más interactivas provocando ideas que ayuden a reforzar los contenidos. Por tal razón, los profesores y el uso que ellos hacen de los recursos tecnológicos en los procesos de enseñanza-aprendizaje constituyen un indicador a tener en cuenta para analizar el avance de la incorporación de las Tic en las universidades los modos en que las TIC están siendo integradas en el currículum, y como son aplicadas en el proceso de enseñanza aprendizaje, tanto por el profesor como por el alumnado; sobre todo en lo que se refiere a la búsqueda y presentación de información.

Respecto a los antecedentes de la integración de las TIC en América Latina se han creado políticas para que se realicen de una manera eficiente en los sistemas de formación inicial docente. Según un estudio realizado por la CEPAL Brun (2011) “(...) participaron 16 países entre Latinoamericanos y del Caribe, los respectivos Ministerios de Educación identificaron los principales desafíos o dificultades actuales y futuras respecto a la inclusión de TIC en FID" (p.16). 
En los países latinoamericanos se tiene en común la insuficiente información o que es difícil tener acceso a ella, asimismo la conectividad deficiente, la capacitación docente escasa o incompleta, pero se están haciendo esfuerzos en las instituciones educativas de incorporar poco a poco la tecnología en el currículo de educación.

Una experiencia que es importante comentar es la de Chile ellos a través del Ministerio de Educación han definido las competencias TIC que deberían presentar los egresados de carreras de pedagogía, las que son divididas en cinco dimensiones:

- Dimensión Pedagógica: demostrar formas de aplicar las TIC en el currículum escolar vigente como una forma de apoyar y expandir el aprendizaje y la enseñanza.

- Dimensión Técnica: dominio de las competencias asociadas al conocimiento general de las TIC y el manejo de las herramientas de productividad (procesador de texto, hoja de cálculo, presentador) e Internet, desarrollando habilidades y destrezas para el aprendizaje permanente de nuevos hardware y software.

- Dimensión Gestión: uso de las TIC para apoyar su trabajo en el área administrativa, tanto a nivel de su gestión docente como de apoyo a la gestión del establecimiento.

- Dimensión Ética, Social y Legal: conocen, se apropian y difunden entre sus estudiantes los aspectos éticos, legales y sociales relacionados con el uso de los recursos informáticos y contenidos disponibles en Internet actuando de manera consciente y responsable respecto de los derechos, cuidados y respetos que deben considerarse en el uso de las tic.

- Dimensión Desarrollo y Responsabilidad Profesional: hacen uso de las TIC como medio de especialización y desarrollo profesional; informándose y accediendo a diversas fuentes para mejorar sus prácticas y facilitando el intercambio de experiencias que contribuyan mediante un proceso de reflexión con diversos actores educativos, para conseguir mejores procesos de enseñanza y aprendizaje. (Ministerio de Educación, 2008).

Por otra parte, es de mencionar las TIC vienen a modificar la forma de enseñar, puesto que existe una variedad de herramientas que se pueden tomar en cuenta para esta finalidad. Por ejemplo para Camacho y Díaz (2012), "Existe una clasificación de acuerdo con el propósito con que se les quiera utilizar, es decir, herramientas digitales para el aprendizaje grupal colaborativo, las herramientas de comunicación: Foros y Chats; asimismo las herramientas de creación de contenido como los: Blogs y Wikis; y las herramientas de aprendizaje social: Twitter, Facebook y YouTube" (p.18). Esta diversidad de recursos en la web facilita el trabajo con los estudiantes en el caso de los Blogs y Wikis se fomenta la búsqueda de información y la creatividad; en el caso de Twitter y Facebook se pueden utilizar como medios de comunicación y formación de grupos de las asignaturas que se están cursando, además de enviarse documentos en el caso de Facebook que permite esta opción. Y por último pero no menos importante YouTube se puede utilizar para inscribirse en canales educativos y hacer uso de videos. En este sentido la Organización de las Naciones Unidas para la Educación, la Ciencia y la Cultura (UNESCO) en el año 2013 realizó un estudio en el que establece que la clave para entender las TIC es que “(...) no son sólo herramientas simples, sino que constituyen sobre todo nuevas conversaciones, estéticas, narrativas, vínculos relacionales, modalidades de construir identidades y perspectivas sobre el mundo" (p.16). Esto hace pensar que los estudiantes se apropien de los usos de estas para mejorar la habilidad de comunicarse con otros pares e intercambiar información educativa.

Por otro lado, la integración de la tecnología en el currículo en Educación Superior exige una valoración de las posibilidades didácticas con los que cuenta. En el Salvador están relacionados, tanto con los recursos que tienen las universidades como con los recursos del gobierno, empresa privada y las organizaciones no gubernamentales.

El divorcio entre la tecnología utilizada por las estructuras gubernamentales o privadas de El Salvador y el nivel tecnológico de las universidades del país son variables que afectan el desarrollo económico y social no solo de El Salvador, sino de la región centroamericana. La incorporación de las Tecnologías de información y comunicación (TIC) es un proceso de aplicación directa en el proceso de enseñanza-aprendizaje. En este sentido Reparaz (2000), propone "la conveniencia de dar primacía y que es preciso tener en cuenta, de cómo las nuevas tecnologías surgen, se desarrollan y someten a continuos y profundos cambios" (p.15), los cuales mejoran el proceso de formación inicial docente, ya que la universidad como principal responsable de la formación debe considerar las exigencias que la sociedad le demanda. A su vez, la integración de las TIC en lo curricular debe estar enfocada en la práctica educativa desarrollada en el contexto de Educación 
Superior. Sin embargo, tal como lo plantea Vaillant (2013) “El conocimiento tecnológico es condición necesaria para avanzar en la integración de las TIC, pero no resulta suficiente para innovar. Los docentes requieren hoy conocimientos pedagógicos sobre el uso de las TIC" (p.7). Respecto a este planteamiento seguramente surge la interrogante ¿Serán las TIC pieza importante para la innovación en el aula? La respuesta es obvia claro que son importantes, pero también es necesario que los docentes sean competentes en el uso de ellas para no caer en el tradicionalismo y siendo el colmo que usando la tecnología en el aula y que a la larga es un error el cual perjudica el proceso de enseñanza-aprendizaje.

En el estudio realizado por la Universidad Tecnológica de El Salvador (UTEC) se obtuvo los siguientes resultados con respecto al uso de la tic en el proceso enseñanza - aprendizaje este refleja resultados positivos, pero más bajos que los resultados en las actitudes. Los docentes usan las TIC en menor medida ya que los resultados son menores, por ejemplo: el $75.2 \%$ de los encuestados manifestaron que utilizan las TIC para optimizar el proceso enseñanza -aprendizaje de manera regular o siempre, el $71.3 \%$ organiza y conduce a los estudiantes en la solución de tareas con herramientas de las TIC, 77.8\% manifiesta que procura la actualización constante en las innovaciones de las TIC y el $82 \%$ utiliza los correos electrónicos para fines académicos (UTEC, 2009).

De tal manera que las competencias digitales en la formación del profesorado asumen, según Pons (2010), citando a la Unión Europea, como: "La capacidad para responder a las exigencias individuales o sociales para realizar una actividad o una tarea" (p.9); por lo que es necesario y urgente desarrollar las competencias digitales básicas en el uso de las TIC en la formación del profesorado, para que estos puedan aplicarlas en su práctica profesional. Así como lo menciona Blanco, Ricoy y Pino (2006), que: "Las TIC permiten a educadores y educandos mejorar el proceso formativo" (p.1211). Desde esta perspectiva, por la sencilla razón que trasciende a mejorar como profesional por la capacidad que posee de hacer uso de recursos tecnológicos para su formación, como lo explica Pons (2010), las competencias deben partir “(...) de una formación que, además de promover el desarrollo de ciertos atributos habilidades, conocimientos, actitudes, aptitudes y valores, considere la necesidad de intervenir dentro del contexto" (p.9). Estos elementos que se describen para la formación de competencias digitales estas consisten en las “(...) habilidades para buscar, obtener, procesar y comunicar información, y transformarla en conocimiento. Además, implica ser una persona autónoma, eficaz, responsable, crítica y reflexiva al seleccionar, tratar y utilizar la información disponible, contrastándola cuando es necesario de acuerdo con la sociedad" (Instituto de tecnologías educativas, 2011, p.2). Esto hace énfasis en su importancia en educación y que se deben considerar el contexto en el que se desarrolla tanto el estudiante en formación como el docente que imparte las asignaturas, ya que debe planificar en qué momento usar las tecnologías y que no se vuelva algo rutinario, por lo tanto, la competencia digital según Cervera (2010), “(...) entraña un reto inherente y específico a ella: la dificultad de su planificación y de su evaluación, la complejidad del diseño del proceso de enseñanza-aprendizaje" (p. 167). Puede ser un reto incorporar al currículo las TIC, no obstante es de tener una actitud positiva especialmente los profesores, ya que al planificar su uso en las clases resultará provechoso para todos los implicados en el proceso educativo.

\section{OBJETIVOS}

\section{Objetivo general}

Analizar cómo los docentes incorporan la tecnología en las clases y en el currículo educativo dentro del nivel de Educación Superior en la carrera académica de Profesorado en Educación Básica para primer y segundo ciclo.

\section{Objetivos específicos}

Apropiar a los estudiantes de la tecnología para mejorar su formación profesional. Identificar los recursos tecnológicos que promueven el cambio educativo en el currículo de la formación inicial docente.

Seleccionar herramientas digitales para generar innovación en los ambientes de enseñanzaaprendizaje de los planes de estudio en el nivel de Educación Básica, implementar la tecnología educativa en el contexto de desempeño de los estudiantes de formación inicial docente.

Verificar las destrezas y habilidades de los estudiantes en el uso de recursos tecnológicos acordes a las características y necesidades de su formación profesional. 


\section{FORMULACIÓN DEL PROBLEMA}

¿Cómo mejorar la incorporación de las tecnologías educativas en los procesos de formación inicial de docentes del nivel de Educación Básica en la Universidad Católica de El Salvador?

\section{DESARROLLO METODOLÓGICO}

La investigación fue de tipo descriptiva-evaluativa, con la finalidad de mostrar cómo en las asignaturas de estudio de la carrera de Profesorado en Educación Básica para primer y segundo ciclo se incorpora la tecnología educativa, la evaluación de profesores y estudiantes con respecto al uso de herramientas tecnológicas, y finalmente, la adquisición de conocimientos adquiridos por los docentes a través de los talleres realizados al inicio de cada ciclo académico.

La población para la investigación fue de 6 estudiantes de la carrera de profesorado del nivel de Educación Básica de primer y segundo ciclo y 12 docentes que impartieron clase a este grupo de estudiantes.

Las técnicas e instrumentos utilizados para la recolección de datos fueron los siguientes:

a. Encuesta: con esta técnica se buscó recopilar información para verificar aspectos de cómo fue la asimilación de la tecnología educativa durante el desarrollo de la carrera profesional. Se utilizó un cuestionario con preguntas abiertas y cerradas, dirigida a los estudiantes de antiguo y nuevo ingreso de la carrera de profesorado.

b. Ficha de revisión de plataforma: este instrumento se implementó para revisar actividades y recursos presentados por el docente en la plataforma virtual Moodle.

c. Entrevista: dirigida a docentes que trabajaron con el grupo de estudiantes de la carrera de profesorado.

Para el análisis e interpretación de la información se hizo a través del método inductivo, observándose los hechos particulares para formar ideas generales de la aplicación de las TIC en el proceso de formación inicial docente.

\section{RESULTADOS}

Este estudio, sirvió para realizar un sondeo de cómo los estudiantes de profesorado en Educación Básica para primer y segundo ciclo asimilaron y aplicaron a lo largo de su carrera la tecnología en Educación. Así mismo, se realizó con la finalidad de conocer los recursos que utilizan frecuentemente en el salón de clases.

Se encontró que Los recursos más utilizados por los docentes son: la computadora, teclado, Central Processing Unit (CPU), cámara fotográfica, mouse, Universal Serial Bus (USB), Compact Disk (CD), proyector multimedia y bocinas. Todos estos les sirven para optimizar procesos educativos y tiempos. Sin embargo, es necesario utilizar otros recursos, por ejemplo Mimio Studio, que es una pizarra digital la cual funciona con un, Ultrasonido infrarrojo con lápiz; esta se trabaja con un dispositivo que se coloca en una pizarra blanca ya existente, ayudada por un lápiz óptico de baterías. Cuando entra en contacto con la superficie de la pizarra, se envía simultáneamente una señal ultrasónica y otra de tipo infrarrojo para establecer sincronía con la computadora. (Medina, 2011, p. 2).

A pesar de que la Facultad de Ciencias y Humanidades cuenta con este equipo, no todos los docentes saben de su existencia y obviamente del uso de este recurso y por consiguiente tampoco los estudiantes. Es necesario, que en la Facultad se hagan talleres en los que se explique el uso del MimioStudio, para que los docentes lo usen en las cátedras causando así novedad e interés de parte de los estudiantes.

Con respecto al acceso de computadora, cinco estudiantes tienen computadora en casa, facilitándoseles el acceso a programas o aplicaciones que han utilizado en clases. Mientras, que solo una estudiante no tiene computadora en casa, pero sí tiene acceso a internet en los ciber café y en la universidad, manifestando que por esa razón se les hace difícil estar al día en lo que corresponde a tecnología.

En cuanto a la identificación de las herramientas digitales que generan innovación en los ambientes de enseñanza-aprendizaje de la formación inicial 
docente del nivel de Educación Básica los docentes entrevistados comentaron que utilizan el portafolio digital y la Web Quest. Estas son herramientas digitales que ayudan a innovar el proceso de enseñanza-aprendizaje porque son dinámicas y eficientes. Estas herramientas se emplearon para impartir clases, y también para que los estudiantes presentaran tareas. Uno de los docentes que formó parte del estudio señaló que en lo que respecta a la Web Quest, solo la conoce de forma teórica; ese detalle hace reflexionar que los docentes deben ser proactivos en su formación con respecto al uso de TIC. Es necesario que ellos sigan explorando opciones digitales para desarrollar sus clases, ya que ellos son los responsables de que la asignatura se actualice constantemente. Por otra parte, todos coincidieron sobre la importancia de utilizar simuladores durante las clases, aunque estos solo se ocupan en el área de Ciencias y Matemáticas.

Los estudiantes durante la carrera académica de profesorado usan más tecnología en la asignatura de Tecnología y Educación porque se les lleva al centro de cómputo. En el programa de esta materia conocieron diferentes software por ejemplo: Microsoft Word, Excel, Publisher, PowerPoint, Paint, por mencionar los más comunes. De igual manera, algunas herramientas digitales como Wix, Cacoo, Prezi, Google Drive, Google Sites, redes sociales, Hot Potatoes y Movie Maker se utilizan para hacer tareas, presentaciones para las clases, compartir información con los compañeros y buscar videos para hacer manualidades. Sin embargo, los estudiantes expresaron que hubiese sido más efectivo ampliar en otras asignaturas el uso de herramientas digitales. Asimismo, opinaron que se debe considerar integrar en la malla curricular de la carrera la tecnología porque es importante dominar está área, especialmente por los cambios que se dan continuamente en la sociedad. Es así como ellos consideran que fue poco lo que aprendieron sobre tecnología en el transcurso de la carrera académica.

Durante el desarrollo del estudio se trabajó con los docentes que impartieron clases a los estudiantes de profesorado de nuevo ingreso, con la finalidad de proporcionar otras herramientas y recursos para que las incorporaran en sus clases. Entre estas los recursos seleccionados que se les dió a conocer a los docentes en los talleres al inicio de cada ciclo académico se desarrollaron: Photo Story, Cmap Tools, Sites Google, Blogger, Prezi y Go Animate. Parte de la investigación fue el monitoreo de los docentes con la finalidad deverificar si estaban aplicando estas herramientas, y efectivamente todos hicieron uso de ellas, esto fue satisfactorio porque al menos con los docentes que se trabajó adquirieron nuevas competencias digitales para aplicarlas con los estudiantes.

Al analizar el aspecto de la tecnología educativa en el contexto de desempeño de los estudiantes de formación inicial docente desde la perspectiva de los catedráticos, la tecnología influye en el desempeño de los estudiantes porque logran tener un mayor acceso a la información. También cabe señalar que otro factor, que influye son las redes sociales, ya que a través de estas pueden compartir documentos con sus compañeros. Uno de los docentes hizo el comentario que en su asignatura le funcionó hacer uso del Facebook. Esta red social es la más popular entre los estudiantes, por lo tanto hay una mayor interacción.

Este dato se pudo constatar porque los estudiantes comentaron que sí utilizan Facebook para mantenerse informados de noticias, subir archivos en el chat, conversar con sus compañeros para coordinar trabajos o tareas de las asignaturas. También, otra red social utilizada por los estudiantes es Twitter, principalmente para mantenerse al tanto de noticias de todo el mundo; y otros que no pertenecen a ninguna red social porque las consideran como pérdida de tiempo. Además, otros solo usan esta red propiamente para entretenimiento. Otra opción es Google+; el cual se utiliza constantemente, y sí se emplea como recurso académico. Sin embargo, los docentes consideran que su utilización no se ve como una obligación.

Al cuestionar a los estudiantes sobre el uso de las redes académicas, se obtuvo como resultado que cinco de ellos las utilizan para compartir información con los compañeros, actualización de información educativa y tecnología; mientras que una estudiante no hace uso de ellas. Dicho sea de paso, los estudiantes comentaron que recibieron influencia positiva de parte de algunos docentes que les impartieron clase, en el sentido que los animaban a usar diferentes herramientas para mejorar las presentaciones de tareas y exposiciones, además de crear sitios como blogs y sites en Google.

Respecto a la verificación de las destrezas y habilidades de los estudiantes en el uso de recursos tecnológicos acordes a las características y necesidades de su formación profesional desde el punto de vista de los docentes, hace falta hacer una inducción en el curso pre-universitario sobre el uso de la plataforma Moodle. Se sugiere esto por la razón 
de que los estudiantes no conocen este recurso, y resta bastante tiempo de la clase estar explicando cómo es el funcionamiento de la misma.

Por otra parte, cuando los estudiantes recién ingresan a la universidad, es fácil detectar quiénes sí tienen conocimientos básicos o avanzados sobre el uso de tecnología, así como también quiénes tienen deficiencias en el uso de las mismas. En vista de ello, un dato importante a mencionar es que durante la observación de clases, entre ellos mismos se ayudaban cuando no podían utilizar algún recurso o herramienta tecnológica, fomentando así el trabajo colaborativo.

Otro dato considerado en la investigación fue el tiempo que los estudiantes hacen uso de las computadoras, este oscila de una hora a seis horas al día. Los alumnos que dedican más tiempo lo hacen porque tienen acceso a este equipo en casa y por lo tanto tienen mayor práctica en el uso de recursos digitales; caso contrario a los que dedican menos tiempo, que se les dificulta practicar lo aprendido en clase.

Por otra parte, los estudiantes consideran que les hace falta mucho por aprender con respecto a la tecnología, ya que cada día es más avanzada. Ellos consideran conocer lo básico en cuanto al manejo de algunos software educativos, computadora y equipo multimedia. Algunos de ellos opinaron que la universidad debería mejorar el tipo de recursos tecnológicos para el uso de los docentes y para ellos, ya que la sociedad demanda profesionales capacitados al $100 \%$ con la tecnología. Por otra parte, una ventaja que tiene el campus universitario es que se cuenta con internet inalámbrico, pero es necesario mejorar el ancho de banda debido a que no tiene buena cobertura. Esta afirmación fue realizada tanto por docentes como por estudiantes.

\section{CONCLUSIONES}

El uso de la tecnología en el ámbito de Educación Superior es importante porque actualmente cuando no se tiene acceso a una biblioteca cercana se puede navegar en la web y encontrar información actualizada, tanto como artículos, libros, videos, simuladores, aplicaciones y otros. Todos estos recursos son útiles en el proceso de enseñanza aprendizaje, especialmente cuando se está en el aula de clase haciendo una presentación de un contenido.
Al identificar los recursos tecnológicos orientados al cambio educativo en el currículo de la formación inicial docente del nivel de Educación Básica, se logró contrastar la realidad en la que se desarrollan las clases en esta carrera en la Universidad Católica de El Salvador, y se infiere que existe una diversidad de herramientas digitales que pueden ser un apoyo para el proceso de enseñanza en las aulas universitarias, solamente que es necesario que en la institución se desarrollen talleres para que los docentes las conozcan y las puedan implementar en su práctica cotidiana con los estudiantes.

\section{RECOMENDACIONES}

Una cantidad significativa de estudios realizados en todo el mundo muestran que el uso de la TIC en el proceso de enseñanza-aprendizaje hace que el estudiante mejore significativamente en su motivación y entendimiento profundo del objeto estudiado, promueven el aprendizaje activo y colaborativo e involucra a los estudiantes en el proceso de aprendizaje continuo a través de toda la vida.

Lo que lleva a pensar que el uso de los recursos debe ser de forma constante en todas las asignaturas con la finalidad de modernizar las clases es necesario, porque las nuevas generaciones de docentes trabajarán con estudiantes que les demandarán actividades innovadoras en esa área. Además, por los continuos cambios que se dan en la sociedad -aunque hay que considerar que no en todas las instituciones públicas se cuenta con centros de cómputo; incluso a lo sumo solo tengan una computadora o un cañón-, es necesario hacer uso de ellos, por lo que los estudiantes tienen que tener la capacidad de conectarlos y usarlos sin ninguna dificultad. Asimismo, es preciso que los estudiantes sean capaces de utilizar eficientemente programas básicos de Microsoft Windows u otras aplicaciones.

En suma de lo anteriormente expuesto, resulta satisfactorio verificar las destrezas y habilidades en el uso de recursos tecnológicos desarrolladas por los estudiantes acordes a las características y necesidades de su formación profesional, ya que ellos consideran necesario prepararse de la mejor manera posible para cuando estén con su grupo de estudiantes en un futuro próximo.

Por otra parte, los formadores de formadores tienen la responsabilidad de brindar sus clases actualizadas, 
utilizando tecnología para salir de lo tradicional e influir en sus estudiantes para que ellos también desarrollen esa cultura vanguardista en sus clases. De este modo, es ineludible fomentar el uso de las redes académicas y sociales con la finalidad de intercambiar información, ya sea con los miembros de su clase o con otras personas del ámbito docente. Los mismos docentes coinciden que usar el Facebook les resulta más factible en algunos aspectos académicos, ya que por naturaleza los estudiantes (nativos digitales), están más pendientes de este tipo de aplicaciones que de la plataforma Moodle. Los Nativos se identifican con la interactividad, es decir, con la respuesta continua a todas y cada una de sus acciones, además de que basan su accionar en la multitarea y los procesos paralelos (cada vez más tareas en lapsos más cortos de tiempo), prefieren los gráficos a los textos, eligen instruirse de manera lúdica y buscan recibir la información de forma ágil y veloz. Utilizan instantáneamente la Web, se conectan desde dispositivos móviles, intercambian mensajes y chatean de forma inmediata. (Mansilla, Pennacchi \& Bricci, 2013, p. 2).

Los formadores de formadores toman en consideración con cuales grupos van a trabajar, es decir, estudiantes que tienen habilidades bastante ágiles para el uso de tecnología y que "Las necesidades emergentes del ejercicio de su tarea como formadores, los invita a reflexionar sobre el uso pedagógico y didáctico de las TIC en los contextos de enseñanza de futuros profesores" (Merodo, Simón \& García, 2012, p.7). Por el mismo contexto que exige más y se necesita de la tecnología para realizar actividades que mejoren el proceso de enseñanza aprendizaje.

\section{CONCLUSIONES}

Finalmente, es de considerar que al aplicar la tecnología en el salón de clase se está innovando y por lo tanto se genera un mayor interés de los estudiantes hacia las asignaturas. Pero también, no se debe abusar en el uso de estas; se debe buscar un equilibrio para lograr el objetivo de generar conocimientos significativos y útiles en los estudiantes. En esta dinámica, la función del docente consiste en generar oportunidades de aprendizaje, en el que el estudiante pueda cuestionarse qué es lo que ya conoce acerca del tema a desarrollar, buscar nueva información, y trabajar en colaboración con otros para resolver problemas y tomar decisiones.

\section{REFERENCIAS}

Argüelles, A. (1998). La Educación Tecnológica en el mundo. D.F. México: Editorial Limusa S.A de C.V.

Belloch, C. (s.f). Las tecnologías de la información y comunicación en el aprendizaje. Recuperado de http://www.uv.es/bellochc/pedagogia/EVA1.pdf

Blanco, E; Ricoy, C., y Pino, M. (2009). Utilización y funcionalidad de los recursos tecnológicos y de las nuevas tecnologías en la educación superior. Red de revistas Científicas de América Latina, el Caribe, España y Portugal (Redalyc). Recuperado de http://redalyc. uaemex.mx/redalyc/pdf/873/87313699014. pdf

Brun, M. (2011). "Las Tecnologías de la Información y la Comunicación en la Formación Inicial Docente en América Latina". CEPAL, División de Desarrollo Social. Serie políticas sociales $N^{\circ}$ 172. Recuperado de http://www.cepal. org/publicaciones/xml/2/44612/Serie_172_ Mario_Brun_TicALIS_09.2011.pdf

Cabero, J. (2007). Nuevas Tecnologías Aplicadas a la Educación. Madrid: España. Ed. Mc Graw Hill.

Cabero. A, J. (2007). Tecnología Educativa. Madrid: España. Ed. McGraw Hill.

Chile, Ministerio de Educación. (2008). Estándares TIC para la Formación Inicial Docente, una Propuesta en el Contexto Chileno. Chile. Ministerio de Educación. Recuperado de http://www.mineduc.cl/

Esparza, M. O. (2011). Consideraciones sobre la implementación de tecnología educativa en América Latina. Revista de Educación y Desarrollo. Recuperado de http://www.eumed.net/rev/ced/28/meo.htm

Fainholc, B. (2004). Lectura crítica en internet, análisis y utilización de los recursos. $1^{\text {a }}$ ed. Ed. Homo Sapiens. Recuperado de http://www. terras.edu.ar/jornadas/88/biblio/88La-Sociedad-de-llnformacion.pdf 
Fernández, P. M. (2001). La aplicación de las nuevas tecnologías en educación. Revista Tendencias pedagógicas No. 6. Recuperado de http://www.tendenciaspedagogicas.com/ revista_numero.asp?_numero=06

García, F. J. (2003). El potencial tecnológico y el ambiente de aprendizaje con recursos tecnológicos: informáticos, comunicativos y de multimedia. Revista electrónica Actualidades investigativas en Educación. Recuperado de http://revista.inie.ucr.ac.cr/uploads/ tx_magazine/potencial.pdf

Instituto de tecnologías educativas, Departamento de proyectos Europeos. (2011). Competencia digital. Recuperado de http://recursostic.educacion.es/blogs/europa/media/blogs/europa/ informes/Competencia_Digital_Europa_ITE_ marzo_2011.pdf

Mansilla, A., Pennacchi, G.V., y Bricci, M.S. (2013). Nativos digitales en la ruralidad: El INTA y el Saber Digital. Recuperado de http: //inta. gob.ar/sites/default/files/script-tmp-inta_ nativos_digitales.pdf

Medina, P. M. (2011). La pizarra digital como recurso educativo. Revista digital innovación y experiencias educativas No. 38. Recuperado de http://www.csi-csif.es/andalucia/modules/mod_ense/revista/pdf/Numero_38/MANUEL\%20JESUS_MEDINA_2.pdf

Merodo, A., Simón, J., y García, M.X. (2012). La incorporación de las TIC en la formación inicial docente. Recuperado de http://fundacionevolucion.org.ar/sitio/wpcontent/ uploads/2013/05/ESP_informe_02.pdf

Nakano, T., Garret, P., Vásquez, A., y Águeda, M. (2014). La integración de las TIC en la Educación Superior: Reflexiones y aprendizajes a partir de la experiencia PUCP. Revista en Blanco \& Negro, Vol. 4, $N^{\circ} 2$. Recuperado de revistas.pucp.edu.pe/index.php/enblancoynegro/article/download/8936/9344
Organización de las Naciones Unidas para la Educación, la Ciencia y la Cultura (UNESCO). (2013). Enfoques estratégicos sobre las TICS en Educación en América Latina y el Caribe. Recuperado de http://www.unEsco.org/new/ fileadmin/MULTIMEDIA/FIELD/Santiago/images/ticsesp.pdf

Reparaz, C; Sobrino, A., y Mir, J. (2000). Integración curricular de las nuevas tecnologías. Barcelona España. Ed. Ariel, S.A.tecnológicos en Educación. Santa Fe: Homo Sapiens.

Scolari, C. A. (2011). Convergencia, medios y educación. Recuperado de http://www.relpe. org/wp-content/uploads/2011/05/Convergencia.pdf

Severin, E. (2011). Competencias para el siglo xxı: cómo medirlas y cómo enseñarlas. Banco Interamericano de Desarrollo (BID). Recuperado de http://idbdocs.iadb.org/wsdocs/ getdocument.aspx?docnum=36239015

Tochero, V. A. (2015). El mito del nativo digital repensando el paradigma Prenskiano. Revista Entre Textos. Año 7, $\mathrm{N}^{\circ}$ 19. Recuperado de http://entretextos.leon.uia.mx/num/19/ PDF/ENT19-11.pdf

Universidad Tecnológica de El Salvador. (2009). Actitudes, dominio y uso de las tecnologías de la información y la comunicación (TIC) de los docentes de las universidades privadas de El Salvador. Recuperado de http: / / www. redicces.org.sv/jspui/bitstream/10972/260/1/56175.pdf

Vaillant, D. (2013). Integración de tic en los sistemas de formación docente inicial y continua para la Educación Básica en América Latina. $1^{\circ}$ ed. Recuperado de https: / /www.unicef.org/ argentina/spanish/educacion_Integracion_ TIC_sistemas_formacion_docente.pdf 